\title{
Further Results on Graceful Digraphs
}

\author{
S. M. Hegde ${ }^{1}$ - Shivarajkumar ${ }^{2}$
}

\begin{abstract}
A digraph $D$ with $p$ vertices and $q$ arcs is labeled by assigning a distinct integer value $g(v)$ from $\{0,1, \ldots, q\}$ to each vertex $v$. The vertex values, in turn, induce a value $g(u, v)$ on each $\operatorname{arc}(u, v)$ where $g(u, v)=(g(v)-g(u))(\bmod q+1)$. If the arc values are all distinct then the labeling is called a graceful labeling of a digraph. In this paper, we prove a general result on graceful digraphs of which Du and Sun's conjecture (J. Beijing Univ. Posts Telecommun, 17: 85-88 1994) is a special case. Further, we provide an upper bound for the number of non isomorphic graceful directed cycles obtained from a graceful labeling of the unicycle $\overrightarrow{C_{n}}$.
\end{abstract}

\section{Introduction}

For standard notations and terminologies in graph theory we follow Chartrand and Lesniak [1].

Graph labelings, where the vertices and arcs are assigned real values or subsets of a set subject to certain conditions, have often been motivated by their utility in various applied fields and their intrinsic mathematical interest (logico-mathematical). Graph labelings were first introduced in the mid sixties. In the intervening years, dozens of graph labeling problems have been studied in over a thousand papers. An enormous body of literature has grown around the subject, especially in the last forty years or so, and is still getting embellished due to an increasing number of application driven concepts [2].

Shivarajkumar

shivarajmacs@gmail.com

S. M. Hegde

smhegde@nitk.ac.in

1 Department of Mathematical and Computational Sciences, National Institute of Technology Karnataka Surathkal, India, Srinivasnagar 575025, India

2 Department of Computer Science \& Automation, Indian Institute of Science, Bangalore 560012, India 
The concept of graceful labeling was introduced by Rosa [3] in the year 1967.

Definition 1 An undirected graph with $e$ edges is gracefully labeled if each vertex $v$ is assigned a distinct value $f(v)$ from the set $\{0,1, \ldots, e\}$ in such a way that the set of edge labels equals $\{1,2, \ldots, e\}$ when edge $u v$ is labeled by $f(u v)=|f(u)-f(v)|$. A graph is said to be a graceful (undirected) graph if it can be gracefully labeled.

The concept of graceful labeling of undirected graphs was extended to digraphs by Bloom and Hsu [4] as follows.

Definition 2 A digraph $D$ with $p$ vertices and $q$ arcs is labeled by assigning a distinct integer value $g(v)$ from $\{0,1, \ldots, q\}$ to each vertex $v$. The vertex values, in turn, induce a value $g(u, v)$ on each $\operatorname{arc}(u, v)$ where $g(u, v)=(g(v)-g(u))(\bmod q+1)$. If the arc values are all distinct then the labeling is called a graceful labeling of a digraph.

Bloom and $\mathrm{Hsu}$ [5] established some connection between graceful digraphs and Latin squares, sequenceable groups, Abelian groups, Galois fields, and neofields. Also they have specified the relation between graceful unicycles and complete mappings by establishing the relation of each to a particular class of permutations.

Definition 3 If both the indegree and outdegree of all vertices of a directed cycle are one, then it is called a unicycle.

Theorem 1 [5] Let $D=\bigcup_{i=1}^{t} \overrightarrow{C_{k_{i}}}$, the union of $t$ disjoint identical unicycles on $n$ vertices. $D$ is graceful if (a) $t=1$ and $n$ is even; of if $(b) t=2$; or if $(c) n=2$ or $n=6$. Moreover, $D$ is not graceful if th is odd.

Definition 4 [6] $n \odot \overrightarrow{C_{m}}$ is the digraph obtained from $n$ copies of the unicycle $\overrightarrow{C_{m}}$ which have one common vertex.

In 1991, Ma Kejie [7] proposed the following:

Conjecture 1 For any even $n, n \odot \overrightarrow{C_{3}}$ is graceful.

In 2000, Jirimutu et al. [8] proved this conjecture. Du and Sun [6] proved that the digraph $n \odot \overrightarrow{C_{2 m}}$ is graceful for every integer $n \geq 1$ and $m \geq 1$, and conjectured that :

Conjecture 2 For any positive even $n$ and any odd $m(m \geq 3)$, the digraph $n \odot \overrightarrow{C_{m}}$ is graceful.

This conjecture has been proved for $m=5,7$ by Jirimutu and Siqinbate [9], $m=9,11$ and 13 by Jirimutu et al. [10], $m=15,17$ by Jirimutu et al. [11], $m=19$ by Bao et al. [12], $m=21$ by Feng et al. [13] and $m=23$ by Bao et al. [14].

A graceful digraph $D$ does not have a unique graceful labeling, since adding a constant modulo $(q+1)$ to all of the vertex labels of a digraph preserves the arc labels and therefore generates a new graceful labeling of $D$. Graceful labeling of the vertices of $D, g_{1}(V(D))$ and $g_{2}(V(D))$ are termed equivalent if $g_{1}(V(D)) \equiv g_{2}(V(D))+k(\bmod q+1)$.

Bloom and Hsu [5] observed the following property for the given digraph which demonstrate how knowing one gracefully labeled digraph is sufficient to determine other graceful digraphs.

Suppose that we reverse the direction of $\operatorname{arcs}(u, v)$ and $(x, y)$ that are labeled respectively by values $k$ and $-k=q+1-k(\bmod q+1)$ to form a digraph $D^{\prime}$. Then in $D^{\prime}, g(v, u)=-k$ and $g(y, x)=k$ and hence the set of vertex labels remains unchanged by this exchange. 
In this paper, we prove a general result of which Conjecture 2 is a special case. Also, we give an upper bound for the number of non isomorphic graceful directed cycles obtained from a graceful labeling of the unicycle $\overrightarrow{C_{n}}$.

\section{Gracefulness of Balanced Digraphs}

In this section, we present the graceful labeling of digraphs using a system of simultaneous congruences. And prove that if a degree balanced digraph $D$ is graceful, then the number of arcs must be even.

Definition 5 [15] A digraph $D$ is said to be balanced if the indegree and outdegree of each vertex is the same.

Theorem 2 If a balanced digraph $D$ is graceful then number of arcs in $D$ is even.

Proof Suppose that $D$ is a graceful balanced digraph with $p$ vertices and $q$ arcs. Let $a_{1}, a_{2}, \ldots, a_{p}$ be the labels of the vertices of $D$.

As $D$ is graceful, corresponding to each $k=1,2, \ldots, q$, we obtain a nonzero element $b_{k}$ of $\mathbb{Z}_{q+1}$ given by:

$$
a_{i}-a_{j} \equiv b_{k}(\bmod q+1)
$$

for some $i, j \in\{1,2, \ldots, p\}$.

As the left hand sides of the system of congruences obtained by a graceful labeling of $D$ add up to zero, the right hand sides also add up to zero. Hence

$$
b_{1}+b_{2}+\ldots+b_{q} \equiv 0(\bmod q+1) .
$$

From the above theorem, it is immediate to see the following lemmas.

Lemma 1 Let $D$ be a graceful digraph with $p$ vertices and $q$ arcs. Suppose that $\vec{C}_{n}$ is a unicycle contained in the digraph $D$. Then the sum of the labels on the arcs of unicycle $\overrightarrow{C_{n}}$ is congruent to zero $(\bmod q+1)$.

Lemma 2 The unicycle $\overrightarrow{C_{n}}$ is graceful if and only if the sum of the elements $1,2, \ldots, n$ is congruent to $0(\bmod n+1)$, and there exists an arrangement of these elements in a circular way, with the sum of $m(m<n)$ consecutive elements not congruent to $0(\bmod n+1)$.

In the view of Lemma 1, the following result proved by Bloom and Hsu [5] becomes a corollary.

Corollary 1 For a union of $n \geq 1$ unicycles to be graceful, it is necessary that the total number of arcs in the digraph be even.

Theorem 3 In a unicycle $\vec{C}_{n}$ ( $n$ is even), if the orientation of the arcs of any unidirectional path is reversed, then the resulting directed cycle is not graceful.

Proof Consider the unicycle $\overrightarrow{C_{n}}$ where $n$ is even. Let us reverse the orientation of the arcs of any unidirectional path $\overrightarrow{P_{j}}$ on $j$ vertices, where $2 \leq j \leq n-1$ of the unicycle $\overrightarrow{C_{n}}$. Assume that the resulting directed cycle is graceful. Let $a_{1}, a_{2}, a_{3}, \ldots, a_{j}, a_{j+1}, \ldots, a_{n-1}, a_{n}$ be the 
labels of the vertices and $b_{1}, b_{2}, \ldots, b_{j}, \ldots, b_{n}$ be the labels on the arcs of the resulting directed cycle.

Then by adding the system of simultaneous congruences representing the graceful labeling of the resulting directed cycle, we get,

$$
2\left(a_{1}-a_{j+1}\right) \equiv 0(\bmod n+1) .
$$

Since the $\operatorname{gcd}(2, n+1)$ is one, we get, $a_{1}-a_{j+1}=0$. This implies $a_{1}=a_{j+1}$, a contradiction. Hence the proof.

\section{Gracefulness of the Digraph $D\left(n_{1}, n_{2}, \ldots, n_{k} ; m_{1}, m_{2}, \ldots, m_{k}\right)$}

In this section, we prove a general result of which Conjecture 2 is a special case.

Definition $6 D\left(n_{1}, n_{2}, \ldots, n_{k} ; m_{1}, m_{2}, \ldots, m_{k}\right)$ where $k$ is any positive integer denotes the digraph having $n_{1}$ unicycles of length $m_{1}, n_{2}$ unicycles of length $m_{2}, \ldots, n_{k}$ unicycles of length $m_{k}$ having one common vertex.

We will be using the following theorem proved by Friedlander et al. [16] in proving Theorem 5.

Theorem 4 If $n \equiv 1(\bmod 6)$, then the nonzero residues $(\bmod n)$ can be partitioned into $(n-1) / 3$ triples such that the elements of each triple have sum congruent to $0(\bmod n)$.

Theorem 5 The digraph $D\left(n_{1}, n_{2}, \ldots, n_{k} ; m_{1}, m_{2}, \ldots, m_{k}\right)$ is graceful if and only if $\sum_{i=1}^{k} n_{i} m_{i}$ is even.

Proof Let $\sum_{i=1}^{k} n_{i} m_{i}=M$. Suppose that the digraph

$D\left(n_{1}, n_{2}, \ldots, n_{k} ; m_{1}, m_{2}, \ldots, m_{k}\right)$ is graceful. Since $D\left(n_{1}, n_{2}, \ldots, n_{k} ; m_{1}, m_{2}, \ldots, m_{k}\right)$ is balanced by Theorem 2, $M$ is even.

Conversely, suppose that, $M$ is even. To prove that the digraph

$D\left(n_{1}, n_{2}, \ldots, n_{k} ; m_{1}, m_{2}, \ldots, m_{k}\right)$ is graceful, we need to prove the following.

1. Sum of the labels on the arcs of each unicycle is congruent to $0(\bmod M+1)$.

2. Sum of the labels on the arcs of any unidirectional path of the digraph $D\left(n_{1}, n_{2}, \ldots, n_{k}\right.$; $\left.m_{1}, m_{2}, \ldots, m_{k}\right)$ is not congruent to $0(\bmod M+1)$.

We give the proof in three Steps:

Step 1. We prove that it is possible to partition $\mathbb{Z}_{M+1} \backslash\{0\}$ into $\sum_{i=1}^{k} n_{i}$ disjoint subsets where:

(i) There are $n_{1}$ subsets each of cardinality $m_{1}, n_{2}$ subsets each of cardinality $m_{2}, \ldots, n_{k}$ subsets each of cardinality $m_{k}$, where $m_{i} \geq 3$ for all $i=1,2, \ldots, k$.

(ii) The sum of the elements of each subset is congruent to $0(\bmod M+1)$.

We note that this is trivial when each $m_{i}$ is even. That is, split the elements from the set $\left\{1,2, \ldots, \frac{M}{2}\right\}$ into $n_{1}$ subsets each of cardinality $\frac{m_{1}}{2}, n_{2}$ subsets each of cardinality $\frac{m_{2}}{2}, \ldots, n_{k}$ subsets each of cardinality $\frac{m_{k}}{2}$ and then adjoin to each of those subsets, the negative of its elements.

If each $m_{i}(1 \leq i \leq k)$ is 3 , then Theorem 4 ensures the existence of a required partition.

Now suppose that some $m_{i}^{\prime} s$ are even and some are odd. Let $m_{1}, m_{2}, \ldots, m_{l} \quad(l \leq k)$ be the cardinalities of the disjoint subsets of $\mathbb{Z}_{M+1} \backslash\{0\}$ having odd number of elements. And let $m_{l+1}, m_{l+2}, \ldots, m_{k}$ be the cardinalities all having even number of elements. 
Now $m_{j}=3+\left(m_{j}-3\right)$, for all $1 \leq j \leq l$ where each $m_{j}-3$ is a non negative even integer. Let $\sum_{j=1}^{l} n_{j}=M_{1}$. Friedlander et al. [16] proved that if $\frac{M_{1}}{2} \equiv 0$ or $1(\bmod 4)$, the nonzero integers in the interval $\left[-3\left(\frac{M_{1}}{2}\right), 3\left(\frac{M_{1}}{2}\right)\right]$ can be partitioned into disjoint subsets, each of cardinality 3, so that the sum of the elements in each set is zero. Also, they proved that if $\frac{M_{1}}{2} \equiv 2$ or $3(\bmod 4)$, the integers $1,2, \ldots, 3\left(\frac{M_{1}}{2}-1\right), 3\left(\frac{M_{1}}{2}+1\right)$ and their negatives can be partitioned into disjoint subsets, each of cardinality 3 , so that the sum of the elements in each set is zero.

Now split the remaining nonzero integers in the interval $\left[-\frac{M}{2}, \frac{M}{2}\right]$ into $n_{1}$ subsets of cardinality $\frac{m_{1}-3}{2}, n_{2}$ subsets of cardinality $\frac{m_{2}-3}{2}, \ldots, n_{l}$ subsets of cardinality $\frac{m_{l}-3}{2}, n_{l+1}$ subsets of cardinality $\frac{m_{l+1}}{2}, n_{l+2}$ subsets of cardinality $\frac{m_{l+2}}{2}, \ldots, n_{k}$ subsets of cardinality $\frac{m_{k}}{2}$. Then adjoin to each of these subsets, the negative of its elements. And to each of the above triples, we adjoin $\frac{m_{1}-3}{2}, \frac{m_{2}-3}{2}, \ldots, \frac{m_{l}-3}{2}$ pairs. This has the effect of decomposing the nonzero integers of $\mathbb{Z}_{M+1} \backslash\{0\}$ into subsets of cardinalities $m_{1}, m_{2}, \ldots, m_{k}$ where the sum of the integers in each set is zero.

In the following steps we prove that the sum of the labels on the arcs of any unidirectional path is not congruent to $0(\bmod M+1)$.

Step 2. Let $\left\{b_{x_{1}}, b_{x_{2}}, \ldots, b_{x_{m_{i}}}\right\}\left(1 \leq i \leq k, 1 \leq x \leq n_{i}\right)$ be the subset of $\mathbb{Z}_{M+1} \backslash\{0\}$ such that the sum of the elements is zero. For any such subset $\left\{b_{x_{1}}, b_{x_{2}}, \ldots, b_{x_{m_{i}}}\right\}$ one can arrange the elements into a cyclic permutation $\left\{b_{x_{1}}, b_{x_{2}}, \ldots, b_{x_{m_{i}}}\right\}$, in such a way that $\sum_{l=j}^{j^{\prime}} b_{x_{l}^{\prime}} \not \equiv$ $0(\bmod M+1)$ for all $\left\{\left(j, j^{\prime}\right): 1 \leq j<m_{i}, j<j^{\prime} \leq m_{i}\right\}$.

Bloom and Hsu [5] proved that the cycle $\overrightarrow{C_{n}}$ is graceful if and only if $n$ is even. This implies that, when $n$ is even, the arrangement of elements $1,2, \ldots, n$ as mentioned in Lemma 2 is always possible. So, without loss of generality one can arrange the elements of any subset of $\mathbb{Z}_{n+1} \backslash\{0\}$ having $m$ elements where $m \leq n$ such that the sum of the $m$ elements is congruent to $0(\bmod n+1)$. This arrangement is in a circular way such that the sum of $r(r<m)$ consecutive elements is not congruent to $0(\bmod n+1)$. Therefore, for any subset $\left\{b_{x_{1}}, b_{x_{2}}, \ldots, b_{x_{m_{i}}}\right\}\left(1 \leq i \leq k, 1 \leq x \leq n_{i}\right)$ one can arrange the elements into a cyclic permutation $\left(b_{x_{1}^{\prime}}, b_{x_{2}^{\prime}}, \ldots, b_{x_{m_{i}}}\right)$, in such a way that $\sum_{l=j}^{j^{\prime}} b_{x_{l}^{\prime}} \not \equiv 0(\bmod M+1)$ for all $\left\{\left(j, j^{\prime}\right): 1 \leq j<m_{i}, j<j^{\prime} \leq m_{i}\right\}$.

Step 3. For any two cyclic permutations say, $\left(b_{x_{1}^{\prime}}, b_{x_{2}^{\prime}}, \ldots, b_{x_{m_{i}}^{\prime}}\right)$ and $\left(b_{y_{1}^{\prime}}, b_{y_{2}^{\prime}}, \ldots, b_{y_{m_{j}}^{\prime}}\right)$ one can arrange the elements in such a way that $\sum_{l=k}^{m_{i}} b_{x_{l}^{\prime}}+\sum_{t=1}^{r} b_{y_{t}^{\prime}} \not \equiv 0(\bmod M+1)$ for $1<k \leq m_{i}, 1 \leq r<m_{j}$.

Since the elements in the above cyclic permutations can be rotated in exactly $m_{i} \times m_{j}$ ways, there are at most $\left(m_{i}-1\right) \times\left(m_{j}-1\right)$ combinations which add up to $0(\bmod M+1)$. If $\sum_{l=k}^{m_{i}} b_{x_{l}^{\prime}}+\sum_{t=1}^{r} b_{y_{t}^{\prime}} \equiv 0(\bmod M+1)$ for some $k$ and $r$, then rotate $\left(b_{x_{1}^{\prime}}, b_{x_{2}^{\prime}}, \ldots, b_{x_{m_{i}}^{\prime}}\right)$ and $\left(b_{y_{1}^{\prime}}, b_{y_{2}^{\prime}}, \ldots, b_{y_{m_{j}}^{\prime}}\right)$ cyclically so that $\sum_{l=k}^{m_{i}} b_{x_{l}^{\prime \prime}}+\sum_{t=1}^{r} b_{y_{t}^{\prime \prime}} \not \equiv 0(\bmod M+1)$ for any $1<k \leq m_{i}, 1 \leq r<m_{j}$.

From Steps 1, 2 and 3, one can see that the digraph $D\left(n_{1}, n_{2}, \ldots, n_{k} ; m_{1}, m_{2}, \ldots, m_{k}\right)$ is graceful.

The following illustration gives the idea of the proof of Theorem 5 .

Illustration. Consider $\mathbb{Z}_{15} \backslash\{0\}=\{-7,-6,-5,-4,-3,-2,-1,1,2,3,4,5,6,7\}$. It is possible to partition $\mathbb{Z}_{15} \backslash\{0\}$ into disjoint subsets of the following cardinalities.

1. $m_{1}=3, n_{1}=2 ; m_{2}=4, n_{2}=2$,

2. $m_{1}=3, n_{1}=3 ; m_{2}=5, n_{2}=1$,

3. $m_{1}=4, n_{1}=1 ; m_{2}=5, n_{2}=2$. 
Fig. 1 A graceful labeling of $D(2,2 ; 3,4)$

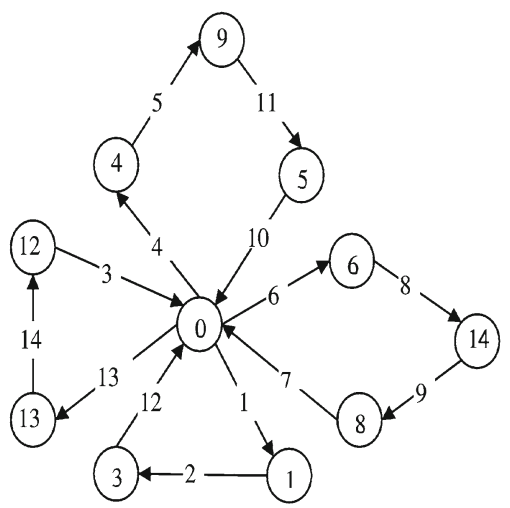

where the sum of elements of each subset is zero.

Since all the other possible partitions can be obtained from the above mentioned partitions, we consider only the above mentioned partitions.

1. Since $n_{1}=2=M_{1}$ and $\frac{M_{1}}{2} \equiv 1(\bmod 4)$ the integers $\{1,2,3\}$ and their negatives can be partitioned into 2 disjoint subsets, each of cardinality 3 , so that the sum of elements in each set is zero. That is, $\{1,2,-3\},\{-1,-2,3\}$. Now split the remaining nonzero integers $\{-7,-6,-5,-4,4,5,6,7\}$ into subsets of cardinalities 2 and 2 . Then adjoin to each of these subsets the negative of its elements. That is, $\{4,5,-4,-5\},\{6,7,-6,-7\}$.

Thus, we have partitioned $\mathbb{Z}_{15} \backslash\{0\}$ into 4 pairwise disjoint subsets of which 2 subsets have cardinality 2 and the other 2 subsets have cardinality 4 such that the sum of the elements in each subset is 0 .

That is,

$$
\{1,2,-3\},\{3,-2,-1\},\{4,-4,5,-5\},\{6,-6,7,-7\} \text {. }
$$

Now we give the arrangement of the subsets as discussed in Step 2.

$$
(1,2,-3),(3,-2,-1),(-4,-5,4,5),(6,-7,-6,7) .
$$

Now we do the arrangement as given in Step 3.

$$
(1,2,-3),(-2,-1,3),(4,5,-4,-5),(6,-7,-6,7) .
$$

Thus, we get a graceful labeling of the digraph $D(2,2 ; 3,4)$. Figure 1 shows a graceful labeling of the digraph $D(2,2 ; 3,4)$.

2. Let $m_{j}=3+\left(m_{j}-3\right)$ where $j=1,2$ and $\sum_{j=1}^{2} n_{j}=4=M_{1}$. Since $\frac{M_{1}}{2} \equiv$ $2(\bmod 4)$ the integers $\{1,2,3,4,5,7\}$ and their negatives can be partitioned into 4 disjoint subsets, each of cardinality 3 , so that the sum of the elements in each set is zero. That is, $\{1,3,-4\},\{2,5,-7\},\{-2,-5,7\},\{-1,-3,4\}$. Then adjoin the remaining pair $\{-6,6\}$ to the triple $\{-1,-3,4\}$. Thus, we have partitioned $\mathbb{Z}_{15} \backslash\{0\}$ into 4 pairwise disjoint subsets of which 3 subsets have cardinality 3 and the other subset has cardinality 5 such that the sum of the elements in each subset is 0 .

That is,

$$
\{1,3,-4\},\{2,5,-7\},\{-2,-5,7\},\{-3,-1,4,-6,6\}
$$


Fig. 2 A graceful labeling of $D(3,1 ; 3,5)$

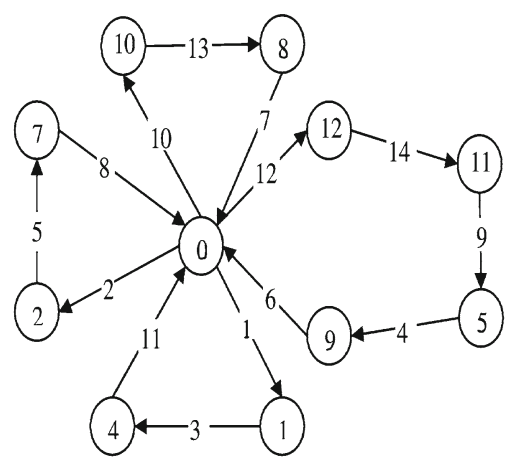

Now we give the arrangement of the subsets as discussed in Step 2.

$$
(1,3,-4),(2,5,-7),(-2,-5,7),(6,-3,-1,-6,4) \text {. }
$$

Now we do the arrangement as given in Step 3.

$$
(1,3,-4),(2,5,-7),(-5,-2,7),(-3,-1,-6,4,6) \text {. }
$$

Thus, we get a graceful labeling of the digraph $D(3,1 ; 3,5)$. Figure 2 shows a graceful labeling of the digraph $D(3,1 ; 3,5)$.

3. Let $m_{2}=3+\left(m_{2}-3\right)$ and $n_{2}=2=M_{1}$. Since $\frac{M_{1}}{2} \equiv 1(\bmod 4)$ the integers $\{1,2,3\}$ and their negatives can be partitioned into 2 disjoint subsets, each of cardinality 3 , so that the sum of the elements in each set is zero. That is, $\{1,2,-3\},\{-2,-1,3\}$.

Now split the remaining non zero integers $\{-7,-6,-5,-4,4,5,6,7\}$ into subsets of cardinalities 2,1 and 1 . Then adjoin to each of these subsets the negative of its elements. That is, $\{-7,-6,6,7\},\{-5,5\}$ and $\{-4,4\}$. Then adjoin the pairs $\{-5,5\}$ and $\{-4,4\}$ to the triples $\{1,2,-3\}$ and $\{-2,-1,3\}$. Thus, we have partitioned $\mathbb{Z}_{15} \backslash\{0\}$ into 3 pairwise disjoint subsets of which 2 subsets have cardinality 5 and the other subset has cardinality 4 such that the sum of the elements in each subset is 0 . That is,

$$
\{1,2,-3,5,-5\},\{-2,-1,3,-4,4\},\{-7,7,-6,6\} \text {. }
$$

Now we give the arrangement of these subsets as discussed in Step 2.

$$
(1,2,5,-3,-5),(-2,-1,-4,3,4),(-7,6,7,-6) \text {. }
$$

Now we do the arrangement as given in Step 3.

$$
(-5,1,2,5,-3),(4,-2,-1,-4,3),(-7,6,7,-6) \text {. }
$$

Thus, we get a graceful labeling of the digraph $D(2,1 ; 5,4)$. Figure 3 shows a graceful labeling of the digraph $D(2,1 ; 5,4)$.

It is easy to see that Conjecture 2 is a special case of Theorem 5.

Corollary 2 [6] For any positive even $n$ and any positive integer $m$, the digraph $n \odot \overrightarrow{C_{m}}$ is graceful. 
Fig. 3 A graceful labeling of $D(2,1 ; 5,4)$

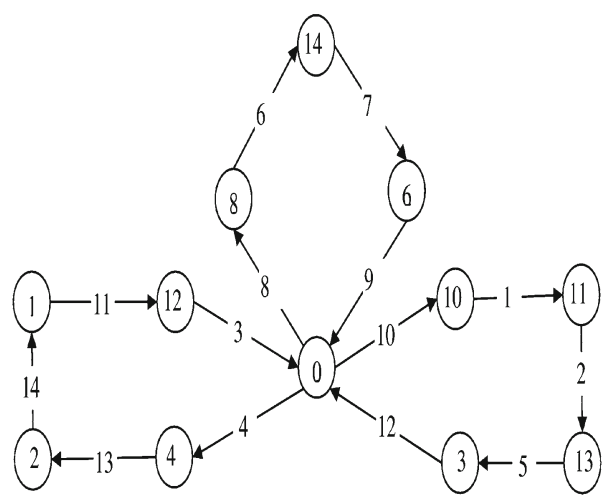

\section{Graceful Labelings of Directed Cycles}

In this section, we provide an upper bound for the number of non isomorphic graceful directed cycles obtained from a graceful labeling of unicycle.

One can see that, in order to obtain the number of non isomorphic graceful directed cycles from a graceful labeling of unicycle, one needs to know the position of the additive inverse pairs on the arcs.

Definition 7 Let $(u, v)=b_{i}$ and $(x, y)=b_{j}$ be any two arcs of the unicycle ${\overrightarrow{C_{n}}}_{n}$. Suppose that $l_{1}$ is the length between $v$ and $x$, and $l_{2}$ is the length of the directed path between $y$ and $u$. Then we define $\min \left\{l_{1}, l_{2}\right\}$ to be the distance between $b_{i}$ and $b_{j}$.

One can see that the maximum distance between any pair of arcs in a unicycle $\overrightarrow{C_{n}}$, where $n$ is even, is $\left(\frac{n}{2}-1\right)$. Hence we get a maximum of $\left(\frac{n}{2}-1\right)$ non isomorphic graceful directed cycles by reversing the direction of a pair of arcs. Let $\left(\frac{n}{2}-1\right)$ be denoted by $a_{1}$.

Similarly, we get $\sum_{i_{1}=1}^{(n / 2-1)} i_{1}, \sum_{i_{2}=2}^{(n / 2-1)} \sum_{i_{1}=1}^{\left(n / 2-i_{2}\right)} i_{1}$ and $\sum_{i_{3}=3}^{(n / 2-1)} \sum_{i_{2}=i_{3}}^{(n / 2-1)} \sum_{i_{1}=1}^{\left(n / 2-i_{2}\right)}$ $i_{1}$ non isomorphic graceful directed cycles after reversing the direction of two, three and four pairs of arcs respectively. Note that in a unicycle $\overrightarrow{C_{n}}$, replacement of $\left\lfloor\frac{n}{4}\right\rfloor+j\left(1 \leq j \leq\left\lfloor\frac{n}{4}\right\rfloor\right)$ pairs of arcs is the same as replacing the remaining $\left\lfloor\frac{n}{4}\right\rfloor-j$ pairs of arcs.

Therefore continuing with the above procedure we get $\sum_{i_{\left\lfloor\frac{n}{4}\right\rfloor}=\left\lfloor\frac{n}{4}\right\rfloor}^{(n / 2-1)} \sum_{i_{\left\lfloor\frac{n}{4}\right\rfloor-1}^{(n / 2-1)}}^{\left(i_{\left\lfloor\frac{n}{4}\right\rfloor}\right.} \ldots$ $\sum_{i_{1}=1}^{\left(n / 2-i_{2}\right)} i_{1}$ non isomorphic graceful directed cycles. Let $a_{2}=\sum_{i_{1}=1}^{(n / 2-1)} i_{1}, a_{3}=\sum_{i_{2}=2}^{(n / 2-1)}$ $\sum_{i_{1}=1}^{\left(n / 2-i_{2}\right)} i_{1}$ and $a_{4}=\sum_{i_{3}=3}^{(n / 2-1)} \sum_{i_{2}=i_{3}}^{(n / 2-1)} \sum_{i_{1}=1}^{\left(n / 2-i_{2}\right)} i_{1}$. Similarly, let $a_{d+4}=\sum_{i_{d+4}=d+4}^{(n / 2-1)}$

$\sum_{\substack{i_{d-3}=i_{d+4} \\ \text { Then }}}^{(n / 2-1)} \ldots \sum_{i_{1}=1}^{\left(n / 2-i_{2}\right)} i_{1}$ for all $d=\left\{1,2, \ldots,\left\lfloor\frac{n}{4}\right\rfloor-4\right\}$.

$$
a_{d+4}=\left\{\sum_{d=1}^{\left\lfloor\frac{n}{4}-4\right\rfloor} \sum_{m=1}^{\frac{n}{2}-d-3} P_{d}(m)\right\} . \sum_{i_{2}=m+d+2}^{\frac{n}{2}-1} \sum_{i_{1}=1}^{\frac{n}{2}-i_{2}} i_{1},
$$

for all $d=\left\{1,2, \ldots,\left\lfloor\frac{n}{4}\right\rfloor-4\right\}$, where $P_{d}(m)=\frac{1}{d !} \prod_{k=0}^{d-1}(m+k)$ denotes the diagonal elements of Pascal's triangle as in [17].

The above discussion leads to the following theorem. 
Table 1 Maximum number of non isomorphic graceful directed cycles of a graceful unicycle $\overrightarrow{C_{n}}$

\begin{tabular}{lc}
\hline$n$ & $\sum_{i=1}^{\lfloor n / 4\rfloor} a_{i}$ \\
\hline 4 & 2 \\
6 & 3 \\
8 & 9 \\
10 & 14 \\
12 & 35 \\
\hline
\end{tabular}

Fig. 4 A graceful labeling of unicycle $\overrightarrow{C_{6}}$

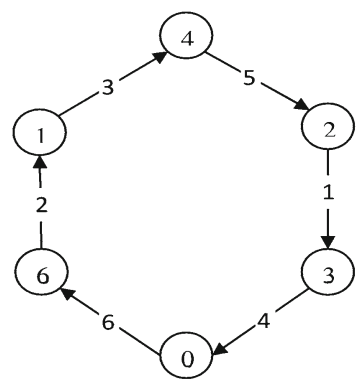

Theorem 6 If a unicycle $\overrightarrow{C_{n}}$ is graceful then it has no more than $\sum_{i=1}^{\lfloor n / 4\rfloor} a_{i}$ non isomorphic graceful directed cycles, each cycle having identically labeled vertices in the common underlying graph $C_{n}$.

Table 1 lists the maximum number of non isomorphic graceful directed cycles obtained from a graceful labeling of unicycle $\overrightarrow{C_{n}}$ for certain values of $n$.

We illustrate the idea of the proof of Theorem 6 with the following example.

Example 1 Consider the unicycle $\vec{C}_{6}$. The cyclic sequence $(6,2,3,5,1,4)$ represents the arc labels of unicycle $\vec{C}_{6}$. Here we can see that $\{1,6\},\{2,5\}$ and $\{3,4\}$ are pairs of additive inverses. The distances between 1 and 6, 2 and 5, 3 and 4 are 1, 1, 2 respectively.

Figure 4 shows a graceful labeling of the unicycle $\vec{C}_{6}$.

Note that in unicycle $\vec{C}_{6}$, replacing a pair of arcs is the same as replacing the remaining two pairs of arcs. Figure 5 shows the non isomorphic graceful directed cycles of a graceful unicycle $\vec{C}_{6}$ by replacing the pairs of arcs labeled 1,6 and 3,4 .

One can see that there are $\left(2^{3}=\right) 8$ non isomorphic directed graphs with $C_{6}$ as its underlying graph. We have already discussed three such directed graphs in Example 1. By Theorem 3 three of the remaining five directed graphs do not admit a graceful labeling. The remaining two directed graphs are graceful displayed in Fig. 6. Note that they cannot be obtained from any of the non equivalent graceful labelings of the unicycle $\vec{C}_{6}$.

Note 1 One can verify that there are 16 and 32 non isomorphic directed cycles with 8 and 10 vertices respectively. We have verified the gracefulness of 12 and 27 directed cycles out of 16 and 32 respectively. From Theorem 3, it follows that the remaining 4 and 5 directed cycles are not graceful. This prompts us to propose the following: 

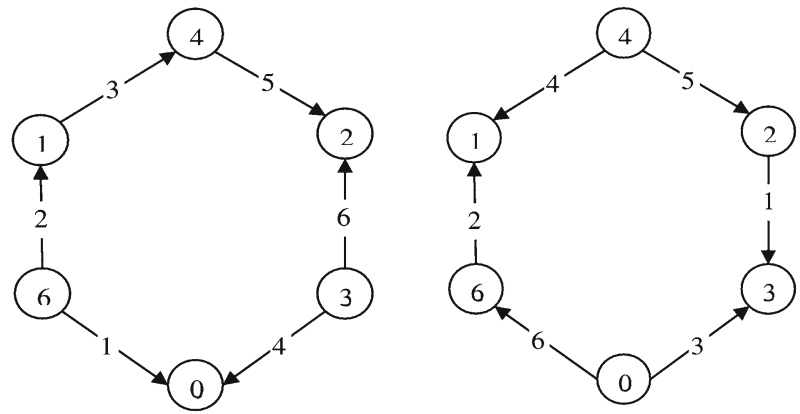

Fig. 5 Graceful directed cycles $\vec{C}_{6}$
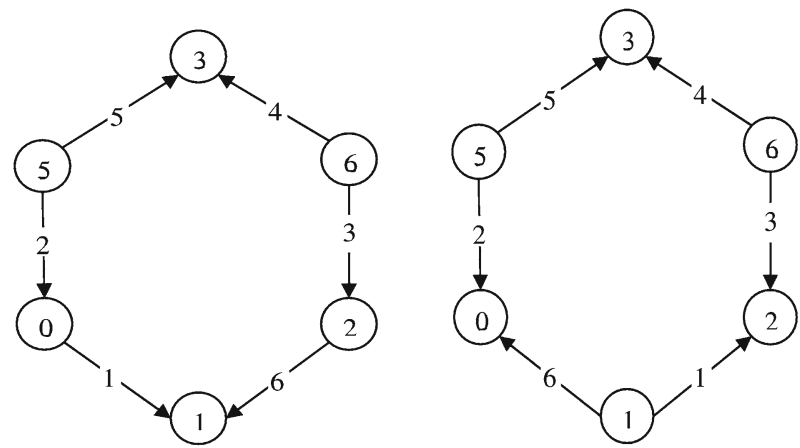

Fig. 6 Graceful directed cycles $\vec{C}_{6}$

Conjecture 3 There exist exactly $2^{\frac{n}{2}}-\frac{n}{2}$ non isomorphic graceful directed cycles with $n$ vertices.

Acknowledgments We would like to thank referees for thier valuable suggestions in the improvement of the paper. Second author would also like to thank Dr. D S Kothari post doctoral fellowship, UGC Govt. of India. The work reported in this paper is a part of the work done under the project No. SR/S4/MS-425/2007 funded by the Department of Science and Technology (DST) Government of India for which we are thankful.

\section{References}

1. Chartrand, G., Lesniak, L.: Graphs and Digraphs. A CRC Press Company, New York (2004)

2. Gallian, J.A.: A dynamic survey of graph labeling. Electron. J. Comb. 16(6), 308 (2013)

3. Rosa, A.: On certain valuations of the verices of a graph. Theory of graphs CED Rosentiel Dunod, Parts 349-355, (1968)

4. Bloom, G.S., Hsu, D.F.: On graceful digraphs and a problem in network addressing. Congressus Numerantium 35, 91-103 (1982)

5. Bloom, G.S., Hsu, D.F.: On graceful directed graphs. Siam. J. Alg. Discr. Methods 6, 519-536 (1985)

6. Du, Z., Sun, H.: $n . \overrightarrow{C_{2 p}}$ are graceful. J. Beijing Univ. Posts Telecommun. 17, 85-88 (1994)

7. Kejie, M.: Graceful Graphs, 1st edn. Beijing University Press, Beijing (1991)

8. Jirimutu, S.: On the proof of a conjecture that the digraph $n \cdot \overrightarrow{C_{3}}$ is a graceful graph. Math. Pract. Theory 30, 232-234 (2000) (in chinese)

9. Jirimutu, S.: On the gracefulness of the digraph $2 k \cdot \vec{C}_{5}$ and $2 k \cdot \vec{C}_{7}$. J. Eng. Math. 16, 134 (1999) 
10. Wang, J., Jirimutu, X.X.: On the gracefulness of the digraph $n . \overrightarrow{C_{m}}$ for $m=9,11$ and 13. Int. J Pure Appl. Math. 23, 393-400 (2005)

11. Wang, J., Jirimutu, X.X.: On the gracefulness of the digraph $n . \overrightarrow{C_{m}}$. J. Inform. Decis. Sci. 23, 1-8 (2009)

12. Bao, Y., Xu, X., Jirimutu, X.: On the gracefulness of the digraph $n . \overrightarrow{C_{m}}$. Int. J Pure Appl. Math. 2, 219-225 (2007)

13. Feng, W., Bao, Y., Jirimutu, X.: On the gracefulness of the digraph $n . \overrightarrow{C_{m}}$ for $m=19$. J. Inform Decis. Sci. 2, 247-252 (2007)

14. Bao, Y., Feng, W., Jirimutu, X.: On the gracefulness of the digraph $n . \overrightarrow{C_{m}}$ for $m=23$. Math. Pract. Theory 38, 213-218 (2008)

15. Richard, A.: Brualdi. Encyclopedia of Mathematics and its Applications. Cambridge University Press, Combinatorial matrix classes, Cambridge (2006)

16. Friedlander, R., Gordon, B., Tannenbaum, P.: Partition of groups and complete mappings. Pacific J. Math. 92, 283-293 (1981)

17. Fowler, D.: The binomial coefficient function. Am. Math. Mon. 103(1), 1-17 (1996) 